Cronfa - Swansea University Open Access Repository

This is an author produced version of a paper published in :

Structural Control and Health Monitoring

Cronfa URL for this paper:

http://cronfa.swan.ac.uk/Record/cronfa33695

\title{
Paper:
}

He, D., Wang, X., Friswell, M. \& Lin, J. (2017). Identification of modal parameters from noisy transient response signals. Structural Control and Health Monitoring, e2019

http://dx.doi.org/10.1002/stc.2019

This article is brought to you by Swansea University. Any person downloading material is agreeing to abide by the terms of the repository licence. Authors are personally responsible for adhering to publisher restrictions or conditions. When uploading content they are required to comply with their publisher agreement and the SHERPA RoMEO database to judge whether or not it is copyright safe to add this version of the paper to this repository. http://www.swansea.ac.uk/iss/researchsupport/cronfa-support/ 


\section{Identification of Modal Parameters from Noisy Transient Response Signals}

\begin{tabular}{|r|l|}
\hline Journal: & Structural Control and Health Monitoring \\
\hline Manuscript ID & STC-16-0220.R1 \\
\hline Wiley - Manuscript type: & Research Article \\
\hline Complete List of Authors: & $\begin{array}{l}\text { He, Dan; Xi'an jiaotong university, school of mechanical engineering } \\
\text { Wang, Xiufeng; Xi'an jiaotong university, school of mechanical engineering } \\
\text { Friswell, Michael I.; Swansea University, School of Engineering } \\
\text { Lin, Jing; Xi'an Jiaotong University, State Key Laboratory for Manufacturing } \\
\text { Systems Engineering }\end{array}$ \\
\hline Keywords: & $\begin{array}{l}\text { Modal identification, Adaptive noise reduction, Low SNR, MMSE-STSA } \\
\text { estimator, WIENER-STSA estimator }\end{array}$ \\
\hline
\end{tabular}

\section{SCHOLARONE ${ }^{\text {m }}$}

Manuscripts 


\section{Identification of Modal Parameters from Noisy Transient}

\section{Response Signals}

3

4

5

6

7

8 School of Mechanical Engineering

9 Xi'an Jiaotong University

10 Xi'an, P R China 
42 Abstract

43

44

In the process of impact testing of large-scale mechanical equipment, the measured forced response signals are often polluted by strong background noise. The forced response signal has a

47 To solve this problem, the mean averaging of repeatedly measured frequency response function 48 estimates is often employed in practical applications. However, a large number of impact testsare 49 not practical for the modal testing of large-scale mechanical equipment. The primary objective of 50 this paper is to reduce the number of averaging operations and improve the accuracy of the modal 51 identification by using an adaptive noise removal technique. An adaptive denoising method is 52 proposed by combining the Wiener and improved minimum mean square error short-time spectral 53 amplitude estimators. The proposed method can adaptively remove both stationary and highly 54 non-stationary noise, while preserving the important features of the true forced response signals.

55 The simulation results show that the proposed noise removal technique improves the accuracy of 56 the estimated modal parameters using only one impulse response signal. The experimental results 57 show that the proposed two step method can accurately identify a natural frequency that is very close to a strong interference frequency in the modal test of a 600MW generator casing. 
63

64

65

66

67

68

69

70

\section{Introduction}

Modal identification estimates the modal model of a structure, i.e. natural frequencies, damping ratios and mode shapes, from measured input-output data. The accuracy of modal identification is highly sensitive to the signal-to-noise ratio (SNR) of the measured output signals(forced response signals). In modal tests of large-scale mechanical equipment, the measured forced response signals are always polluted by strong background noise, and the noise is rather complex as the contributing factors are diverse and complicated. The noise sources are thought to originate from test environment including non-linear effects, extraneous structural noise as well as 'noise' in electronic devices[1]. Hence, the forced response signals have a low SNR and this makes the estimation of the modal parameters difficult. To obtain the ideal forced responses the background noise should be removed from the measured forced response signals. Denoising methods have been proposed for noise removal from frequency response functions(FRFs). Kim and Hong [2] proposed a robust wavelet denoising method for FRFs estimation, which is based on a wavelet-related median filtering and wavelet shrinkage to reduce the effect of outliers and zero-mean Gaussian noise respectively. But the method requires many averaging operations for accurate FRF estimation, which reduces the scope of its application. Sanliturk and Cakar [1] presented a method based on the singular value decomposition(SVD) for the elimination of noise from measured FRFs so as to improve the accuracy of modal identification, but the method needs to set an appropriate threshold to avoid loss of valuable information. Alamdari et al. [3] introduced a Gaussian kernel algorithm to reduce unnecessary noise from noisy FRFs, and it is designed to localize damage in the presence of heavy noise influences by using FRFs of the damaged structure only. Huet al. and Bao et al.[4,5] introduced a Cadzow's algorithm to reduce unnecessary noise from noisy FRFs, but the denoising method needs to set a reasonable noise threshold based on the measured signals. The effectiveness of the denoising methods in [3-5] was illustrated by simulation and experimental data, but none of the results show that the two denoising methods can remove strong background noise mixed in a forced response signal.

Insert Figure 1 here 
Figure 1 shows the basic breakdown of signals into different types[6,7]. The most fundamental division is into stationary and non-stationary signals. Stationary signals have statistical properties that are invariant with time, whereas for non-stationary signals the statistical properties vary with time. Figure 1 highlights that typical measured forced response signals contain both stationary and non-stationary components for modal tests on large-scale mechanical equipment. The ideal forced response signal has a transient component that is non-stationary, background noise that is mainly stationary, and often a continuous component that is non-stationary. The existing denoising algorithms cannot remove stationary noise and non-stationary noise simultaneously, and these different types of noise should be dealt with separately. The short-time spectral amplitude(STSA), Wiener filter(WIENER) and minimum mean square error(MMSE) methods have been widely used in denoising and coding [8-15]. The MMSE-STSA estimator is effective in removing stationary signals and the continuous components of non-stationary signals from measured speech signals[8], although the technique requires the SNR a priori. Hence this SNR is a key parameter in the MMSE-STSA estimator. The decision-directed(DD) approach[8] is a widely used method to estimate the a priori SNR, but has two inherent drawbacks:

- The estimated a priori SNR is biased since the DD approach depends on the estimate of the spectrum in the previous window $[8,9]$.

- The estimated a priori SNR is distorted when the measured signal has a low SNR[8].

The first problem has been solved by an improved a priori SNR estimation method proposed by Plapouset et al.[9], which removes the bias in the DD approach. However, the second problem is still unsolved and hence the MMSE-STSA estimator method cannot be directly used to remove strong background noise mixed in a forced response signal. The Wiener filter is an optimal method to remove stationary noise in stationary environments[16], whereas the Wiener short-time spectral amplitude estimator (WIENER-STSA)improves the application scope of the Wiener filter. Here, the WIENER-STSA estimator can be used to eliminate stationary noise from the measured forced response signal, so as to solve the second problem. In this paper, we propose an adaptive denoising method combining WIENER-STSA and MMSE-STSA estimators with improved a priori SNR estimation. The proposed denoising method can adaptively remove stationary noise 
121 and continuous components of non-stationary noise, while preserving the important features of the

122 true forced response signals. The proposed method can reduce the number of averaging operations

123 and improve the accuracy of modal identification for low SNR measurements.

124 The paper is organized as follows. Section 2 introduces some background about denoising, and 125 compares two a priori SNR estimation methods. Section 3 introduces the proposed method. In 126 section 4, the proposed method is validated using simulated signals. Section 5 applies the 127 proposed method to measured forced response signals collected from a 600MW generator. Finally, 128 conclusion are given in Section 6.

\section{Background}

131

\subsection{The MMSE-STSA estimator}

Ephraim and Malah [8] proposed the minimum mean-square error short-time spectral amplitude(MMSE-STSA) estimator. Previous studies[8, 17] have shown that the MMSE-STSA estimator has a beneficial effect for the processing of non-stationary signals when the SNR level is high. Here the MMSE-STSA developed in [8] is reviewed.

In the usual additive noise model, the measured impulse response signal is given by

$$
x(t)=s(t)+n(t), 0 \leq t \leq T(1)
$$

where $s(t)$ and $n(t)$ denote the noise-free impulse response signal and the noise signal, respectively, in the analysis interval $[0, T]$. Applying the Short Time Fourier Transform (STFT), we have

$$
X_{k}(p)=S_{k}(p)+N_{k}(p)
$$

where $p$ and $k$ denotethe short-time window and the frequency indices, respectively. Using exponential notation, the $k$-th spectralcomponent ofthe noise-free impulse response signal and the noisy signal can be expressed as $S_{k}(p)=A_{k} e^{j \alpha_{k}}$ and $X_{k}(p)=R_{k} e^{j v_{k}}$, respectively[8,9].

The objective of the MMSE-STSA estimator is to determine $\hat{A}_{k}$, the estimate of the spectral amplitude $A_{k}$ of the noise-free impulse response signals $(t)$. Ephraim and Malah [8] estimated $\hat{A}_{k}$ through the minimization of a Bayesian cost function which measures the mean square error between $\hat{A}_{k}$ and $A_{k}$. Thus the Bayesian cost function can be expressed as: 


$$
J=\mathbf{E}\left\{\left(A_{k}-\hat{A}_{k}\right)^{2}\right\}(3)
$$

150

152

153

154

155

156

157

158

159

160

161

162

163

164

165

166

167

168

169

170

171 where $\Gamma(\cdot)$ is the gamma function, with $\Gamma(1.5)=\frac{\sqrt{\pi}}{2}, M(a ; b ; c)$ is the confluent hyper geometric

172 function, and $S N R_{\text {prior }}(k)$ and $S N R_{\text {post }}(k)$ represent the a priori Signal-to-Noise Ratio (SNR)

173

where $\mathbf{E}\{\cdot\}$ is the expectation operator. The Bayesian estimator is then given by

$$
\hat{A}_{k}=\mathbf{E}\left\{A_{k} \mid x(t), 0 \leq t \leq T\right\}(4)
$$

Assuming the individual spectral components are statistically independent of one another, the expected value of $A_{k}$ given $\{x(t), 0 \leq t \leq T\}$ is equal to the expected value of $A_{k}$ given $X_{k}$ only.

We therefore have

$$
\hat{A}_{k}=\mathbf{E}\left\{A_{k} \mid X_{k}\right\}=\frac{\int_{0}^{\infty} \int_{0}^{2 \pi} a_{k} p\left(X_{k} \mid a_{k}, \alpha_{k}\right) p\left(a_{k}, \alpha_{k}\right) d a_{k} d \alpha_{k}}{\int_{0}^{\infty} \int_{0}^{2 \pi} p\left(X_{k} \mid a_{k}, \alpha_{k}\right) p\left(a_{k}, \alpha_{k}\right) d a_{k} d \alpha_{k}}(5)
$$

where the symbol $a_{k}$ denotes the sample value of $A_{k}$, and $p(\cdot)$ denotes a probability density function(PDF). In order to develop the theory along the lines that it has been done in the past it is necessary to treat the Discrete Fourier Transform(DFT) coefficients as Gaussian distributions, the assumption is quite poor in some cases but it appears that the resulting algorithm can still provide useful results. With the Gaussian distribution assumption of each individual spectral component of the noise-free impulse response signal and the noisy signal, the conditional PDF of the observed spectral component given $a_{k}$ and $\alpha_{k}, p\left(X_{k} \mid a_{k}, \alpha_{k}\right)$, is given by

$$
p\left(X_{k} \mid a_{k}, \alpha_{k}\right)=\frac{1}{\pi \lambda_{n}(k)} \exp \left\{-\frac{1}{\lambda_{n}(k)}\left|X_{k}-a_{k} \exp \left(j \alpha_{k}\right)\right|^{2}\right\}(6)
$$

and the joint PDF of the impulse response signal spectral amplitude, $p\left(a_{k}, \alpha_{k}\right)$, is given by

$$
p\left(a_{k}, \alpha_{k}\right)=\frac{a_{k}}{\pi \lambda_{s}(k)} \exp \left\{-\frac{a_{k}^{2}}{\lambda_{s}(k)}\right\}(7)
$$

where $\lambda_{n}(k) \triangleq \mathrm{E}\left\{\left|N_{k}\right|^{2}\right\}$ and $\lambda_{s}(k) \triangleq \mathrm{E}\left\{\left|S_{k}\right|^{2}\right\}$ are the variance of the $k$-th spectral component of the noisy signal and the noise-free impulse response signal, respectively. Substituting Eqs. (6) and (7) into Eq. (5), the MMSE-STSA estimator of the impulse response signal spectral amplitude is obtained as

$$
\hat{A}_{k}=\sqrt{\left[\frac{1}{\lambda_{s}(k)}+\frac{1}{\lambda_{n}(k)}\right]^{-1}} \cdot \Gamma(1.5) \cdot M\left(-0.5 ; 1 ;-\frac{S N R_{\text {prior }}(k)}{1+S N R_{\text {prior }}(k)} S N R_{\text {post }}(k)\right) \cdot A_{k}(8)
$$




$$
\begin{gathered}
S N R_{\text {post }}(k)=\frac{\left|X_{k}\right|^{2}}{\mathrm{E}\left\{\left|N_{k}\right|^{2}\right\}}(9) \\
S N R_{\text {prior }}(k)=\frac{\mathrm{E}\left\{\left|S_{k}\right|^{2}\right\}}{\mathrm{E}\left\{\left|N_{k}\right|^{2}\right\}} .(10)
\end{gathered}
$$

Finally, applying the inverse STFT operation and the phase information of the measured signal, the estimator of the noise-free impulse response signal can be obtained. In practical implementations of the MMSE-STSA estimator, $\mathrm{E}\left\{\left|S_{k}\right|^{2}\right\}$ and $\mathrm{E}\left\{\left|N_{k}\right|^{2}\right\}$ are unknown since only the measured signal spectrum $X_{k}$ is available. Thus, both $\mathrm{E}\left\{\left|S_{k}\right|^{2}\right\}$ and $\mathrm{E}\left\{\left|N_{k}\right|^{2}\right\}$ have to be estimated. In practice, $\mathrm{E}\left\{\left|N_{k}\right|^{2}\right\}$ can be easily estimated during pauses in the impulse response using a classic recursive relation [17], continuously using Minimum Statistics [18] or Minima Controlled Recursive Averaging [19], whereas the priori SNR is a key parameter in the MMSE-STSA estimator. The estimation of the priori SNR will be discussed in detail in the following sections.

\subsection{The a priori SNR estimation method}

A widely used method to determine the a priori SNR from distorted speech is the decision-directed(DD) approach. Ephraim and Malah [8] defined the DD approach as a linear combination of the a posteriori SNR and the instantaneous SNR, with a weighting parameter, $\beta$, that is constrained to be $0<\beta<1$. The linear combination gives

$$
S \widehat{N} R_{\text {prior }}^{D D}(p, k)=\beta \frac{|\hat{S}(p-1, k)|^{2}}{\widehat{\gamma}_{n}(p, k)}+(1-\beta) \mathrm{P}\left[S \widehat{N} R_{\text {post }}(p, k)-1\right](11)
$$

where $p$ and $k$ denote the short-time window and frequency indices, respectively, $\mathrm{P}[x]=x$ if $x \geq 0$ and $\mathrm{P}[x]=0$ otherwise. The parameter $\beta$ is set to a typical value of 0.98 for the DD approach. However, Plapous et al. [9] showed that the DD algorithm introduces a window delay when the parameter $\beta$ is close to one, and this delay introduces a bias in the SNR estimation. Consequently, the DD algorithm computed at the current window $p$ matches that at the previous window $p-1$. Thus, Plapous et al. [9] proposed to compute the SNR for the next window $p+1$ using the DD approach and toapply it to the current window because of the window delay. Hence, an improved a priori SNR estimation method is

$$
S \widehat{N} R_{\text {prior }}^{T S N R}(p, k)=\beta \frac{\left|G_{m m s e}^{D D}(p, k) X(p, k)\right|^{2}}{\widehat{\gamma}_{n}(p, k)}+(1-\beta) \mathrm{P}\left[S \widehat{N} R_{\text {post }}(p+1, k)-1\right](12)
$$


201 The improved a priori SNR estimation method solves the bias problem while maintaining the

202

203

204

205

206

207

208

209

210

211

212

213

214

215

216

217

218

219

220

221

222

223

224

225

226

227

228

229 benefits of the DD approach [9]. In order to measure the performance of SNR estimators, it is useful to compare the estimated SNR values to the true(actual) ones, as shown in Figure 2 where the estimated SNRs are displayed versus the true SNRs. The SNRs are plotted for a simulated signal(to be described in detail in Section 4) to focus the analysis on the behavior of the SNR estimators for forced response components.

Insert Figure 2 here

Figure 2 compares the actual SNR versus the estimated SNRs using the posteriori algorithm, the improved algorithm and the DD algorithm given by Eqs.(9), (11) and (12), respectively. In this case, the solid line corresponds to the actual SNR that can be used as a reference. From Figure 2, it is obvious that the a priori SNR estimator based on the improved algorithm is closer to the actual SNR than the a priori SNR estimator based on the DD algorithm at higher SNR levels. However, the a priori SNR estimator based on the improved algorithm departs from the true SNR at lower SNR levels. The improved algorithm is superior to the traditional DD algorithm when the measured impulse response signal has a higher SNR, but is distorted when the measured impulse response signal has a low SNR. In order to avoid the low SNR situation, the WIENER-STSA estimator will be introduced to improve the SNR.

\subsection{The WIENER-STSA estimator}

The Wiener filter is an optimal method to remove stationary noise in stationary environments [16]. Here, the WIENER-STSA estimator is introduced to enhance the application scope of the Wiener filter. Adopting the noise model mentioned in Section2.1, we assume $s(t)$ and $n(t)$ to be uncorrelated stationary random process, with power spectral density functions denoted by $S_{s}(k)$ and $S_{n}(k)$ respectively, where $k$ denotes the frequency index. One approach to recover the desired signals $(t)$ relies on the additivity of power spectra

$$
S_{x}(k)=S_{s}(k)+S_{n}(k)(13)
$$

To recover a sequence $s(t)$ corrupted by additive noise $n(t)$, that is from the sequence 

$x(t)=s(t)+n(t)$, a linear filter $h(t)$ is found, such that the sequence $\hat{s}(t)=h(t) * x(t)$

231 minimizes the expected value of the nosie, under the condition that the signals $s(t)$ and $n(t)$ are stationary and uncorrelated. The frequency domain solution to this stochastic optimization problem is given by

$$
H(k)=\frac{S_{S}(k)}{S_{S}(k)+S_{n}(k)}(14)
$$

which is referred as the Wiener filter. Since the Wiener filter is derived under uncorrelated and stationary conditions, the Wiener filter provides noise suppression without significant distortion in the estimated signal and the background residual. The STFT is applied when the background and desired signals are non-stationary, and then $S_{s}(k)$ and $S_{n}(k)$ can be expressed as time varying functions $S_{s}(p, k)$ and $S_{n}(p, k)$, where $p$ represents the short-time window. Thus every time window is processed by a different Wiener filter, defined as

$$
H(p, k)=\frac{\hat{S}_{S}(p, k)}{\hat{S}_{S}(p, k)+\hat{S}_{n}(p, k)}=\frac{\frac{\hat{S}_{S}(p, k)}{\widehat{S}_{n}(p, k)}}{1+\frac{\hat{S}_{S}(p, k)}{\hat{S}_{n}(p, k)}}=\frac{\operatorname{SN} \mathrm{R}(p, k)}{1+\operatorname{SN} \mathrm{R}(p, k)}(15)
$$

The reduction of the noise is based on obtaining an accurate SNR [20]. In order to effectively remove stationary noise, an instantaneous SNR will be introduced, defined as

$$
S N R_{\text {inst }}(p, k)=\frac{|X(p, k)|^{2}-\mathrm{E}\left[|N(p, k)|^{2}\right]}{\mathrm{E}\left[|N(p, k)|^{2}\right]}(16)
$$

where $X(p, k)$ is available, and the estimators of $\mathrm{E}\left[|N(p, k)|^{2}\right]$ have been introduced in Section 2.1. Hence, the WIENER-STSA estimator is obtained as

$$
\hat{S}(p, k)=H(p, k) \cdot X(p, k)=\frac{S N R_{\text {inst }}(p, k)}{1+S N R_{\text {inst }}(p, k)} \cdot X(p, k) .(17)
$$

Finally, applying the inverse STFT operation and the phase information of the measured signal, the estimator of the noise-free impulse response signal can be obtained.

\section{Proposed Method}

We assume that the ideal forced response signal is the transient component of non-stationary signal, and the background noise has a stationary component and a continuous component that is non-stationary. An adaptive denoising method is proposed to obtain the ideal forced response 
255 signal. In the first step, the WIENER-STSA estimator is used to remove the stationary signal

256 components, which is very helpful in improving the SNR of the measured forced response signals

257 and make the filtered signals suitable for further processing. In the second step, the MMSE-STSA

258 estimator with an improved a priori SNR estimation method is introduced, which can be used to

259 remove the continuous component of the non-stationary signal. The flow chart of the proposed

260 method is shown in Figure 3.

261

262

263

264

265

266

267

268

269

270

271

272

273

274

275

276

277

278

279

280

281

282

Insert Figure 3 here

The implementation of the proposed denoising method is summarized below:

(1) Estimate the noise PSD $\mathrm{E}\left[|N(p, k)|^{2}\right]$ during no forced response using the Minima Controlled Recursive Averaging approach [19].

(2) Calculate the instantaneous SNR using Eq. (16).

(3) Remove stationary noise components from the measured forced response signal using the WIENER-STSA estimator with an instantaneous SNR estimation method.

(4) Re-estimate the noise PSD $\mathrm{E}\left[|N(p, k)|^{2}\right]$ during no forced response using the Minima Controlled Recursive Averaging approach [19].

(5) Calculate the improved a priori SNR using Eq. (12).

(6) Remove residual non-stationary noise from the filtered forced response signals using the MMSE-STSA estimator with the improved a priori SNR estimation method.

In this paper, the a priori SNR estimation always uses the improved a priori SNR estimation method, and the following parameters have been chosen: short-time window $p=0.06$, windows overlap $50 \%$ and weighting parameter $\beta=0.98$.

\section{Simulated Example}

To validate the proposed method, a simulated signal, $x(t)$, is generated according to the model 


$$
\begin{aligned}
x(t)= & \sum_{i=T}\left(\begin{array}{l}
s_{1} \cdot e^{-\zeta_{1}(t-i)} \cdot \cos \left(2 \pi f_{n 1}(t-i)\right)+s_{2} \cdot e^{-\zeta_{1}(t-i)} \cdot \cos \left(2 \pi f_{n 2}(t-i)\right) \\
+s_{3} \cdot e^{-\zeta_{1}(t-i)} \cdot \cos \left(2 \pi f_{n 3}(t-i)\right)+s_{4} \cdot e^{-\zeta_{2}(t-i)} \cdot \cos \left(2 \pi f_{n 4}(t-i)\right)
\end{array}\right) \\
& +\left(\left(s_{5} \cdot \cos \left(2 \pi f_{r 1} t\right)\right) \cdot\left(s_{6} \cdot \cos \left(2 \pi f_{r 2} t\right)+s_{7} \cdot \cos \left(2 \pi f_{r 3} t\right)+1\right)\right) \cdot \text { random noise }(14)
\end{aligned}
$$

284

285

286

287

288

289

290

291

292

293

294

295

296

297

298

299

300

301

302

303

304

305

306

307

308

309

310
The parameters of the simulated signal are given in Tables 1 and 2, and the sampling frequency is $1024 \mathrm{~Hz}$.

Insert Table 1 here

Insert Table 2 here

The simulated signal, $x(t)$, is composed of two terms. The first term represents a forced response signal, where $s_{1}, s_{2}, s_{3}$ and $s_{4}$ are the amplitudes of the impulse response signal, $f_{n 1}$, $f_{n 2}, f_{n 3}$ and $f_{n 4}$ are the corresponding natural frequencies and $i$ is the sample time increment. The second term represents noise components. According to the mathematical model and the parameters the simulated signal has the following three characteristics.

(1) The forced response signal has a low $\mathrm{SNR}(\mathrm{SNR}=-4.6 \mathrm{~dB})$.

(2) The noise components contain stationary noise and non-stationary noise.

(3) The noise components contain a base frequency $\left(f_{r 1}\right)$, which is very close to a natural frequency $\left(f_{n 2}\right)$ and makes it difficult to accurately estimate the natural frequency $\left(f_{n 2}\right)$.

The simulated signal $x(t)$ is shown in Figure 4; the simulated signal contains significant environmental noise, and the forced response signal has a low SNR. The proposed denoising method was applied to the simulated signal, and the results are shown in Figure 5 and Figure 6. Figure 5 compares the filtered signals from the MMSE-STSA and the proposed methods in the time domain, and Figure 6 compares the results in the frequency domain. Figure 6 shows that the natural frequencies cannot be accurately estimated using the raw simulated forced response signal spectrum.

Insert Figure 4 here

Insert Figure 5 here

Insert Figure 6 here 
312 Figure 5 shows that both the MMSE-STSA and the proposed method can remove most of the 313 environmental noise. The zoomed part of Figure 5 shows that the filtered signal with only the 314 MMSE-STSA method is distorted in the time domain. According to the simulated signal 315 parameters, the first two true natural frequencies are $44.0 \mathrm{~Hz}$ and $50.0 \mathrm{~Hz}$; however Figure 6 shows 316 that the filtered first two natural frequencies using the MMSE-STSA method are predicted to be $31742.0 \mathrm{~Hz}$ and $49.5 \mathrm{~Hz}$. Figure 5 and Figure 6 show that the filtered signal with the proposed method 318 has a good consistency with the ideal forced response signal in both the time and frequency 319 domains. Meanwhile, the strong colored noise frequency $(49.5 \mathrm{~Hz})$ disappears after the filter 320 operation of the proposed method, and the two close natural frequencies $(44.0 \mathrm{~Hz}, 50.0 \mathrm{~Hz})$ are accurately estimated. The simulation results indicates that using the WIENER-STSA estimator before the MMSE-STSA estimator under low SNR conditions significantly improves the estimation. The proposed noise removal technique can improve the accuracy of the estimated modal parameters using only one impulse response signal in a strong background noise environment.

\section{Experiment Results from a 600MW Generator}

\subsection{Experimental setup}

In this section, the proposed method is validated using the measured forced response signals collected from a 600MW generator. The generator exhibits excessive vibration during operation, and the rotating frequency of the generator is $50 \mathrm{~Hz}$. Figure 7 shows the image of the generator, and the generator shell located inside a sound-proof housing. Figure 8 shows the bode diagram of the generator; the generator has a resonance frequency at $48.5 \mathrm{~Hz}$, which is not the natural frequency of the rotor according to the simulated results. Hence, this resonance frequency is likely 
frequency at $48.5 \mathrm{~Hz}$. This makes it difficult to accurately estimate the natural frequency of the generator shell. It should be mentioned that the state-of-the-art method (PolyMAX algorithm) estimates the natural frequency to be $49.8 \mathrm{~Hz}$, which indicates that the strong colored noise signal has a great influence on modal parameter identification.

Insert Figure 7 here

Insert Figure 8 here

347

\subsection{Experimental results}

349

The time domain waveform of the measured signal is shown in Figure 9.The measured signal contains high levels of environmental noise, and the forced response signal has a low SNR. The proposed denoising method was applied to the measured forced response signal, and the results are shown in the time and frequency domains in Figure 10 and Figure 11 respectively. The filtered signal from the MMSE-STSA and the proposed methods are compared. Figure 11 shows that the natural frequencies cannot be accurately estimated from the raw measured forced response signal spectrum. Figure 10 shows that most of the environmental noise has been removed by both the MMSE-STSA and proposed methods. Figure 11 shows that the colored noise frequency $(49.8 \mathrm{~Hz})$ has been filtered using both the MMSE-STSA method and the proposed method, but a natural frequency close to the interference frequency $(49.8 \mathrm{~Hz})$ disappears with the MMSE-STSA method. This demonstrates that the MMSE-STSA method may lead to distortion of the estimated modal parameters in noisy environments. In contrast, Figure 11 shows that a natural frequency at $48.9 \mathrm{~Hz}$ appears after using the proposed method, and the result is consistent with the Bode diagram during the rotor startup. Thus, the application of the WIENER-STSA estimator is necessary before the MMSE-STSA estimator under low SNR conditions, and the proposed method can help to accurately identify natural frequencies in modal tests of large-scale mechanical equipment.

Insert Figure 9 here

367 Insert Figure 10 here

Insert Figure 11 here 
370

371 In this paper, we focus on modal parameter identification when the forced response signal has a

372 low SNR. An adaptive denoising method based on the WIENER-STSA estimator and an improved

373 MMSE-STSA estimator was proposed. Comparing the proposed method with some

374 state-of-the-art denoising methods in Ref. [1,2], the proposed method does not need to set an

375 appropriate threshold to avoid loss of valuable information, and does not require many averaging

376 operations. The proposed method can adaptively remove stationary and non-stationary noise

377 components, while preserving the important features of the true forced response signals. The

378

379

380

381

382

383

384

385

386

387

388

389

390

391

392 simulation shows that the proposed noise removal technique improves the accuracy of the estimated modal parameters using only one impulse response signal, which demonstrates that the proposed method can reduce the number of averaging operations when the measured forced response signal has a low SNR. In the modal test of a 600MW generator shell, the measurement results show that, in contrast to the state-of-the-art method (PolyMAX algorithm), the proposed method can accurately identify a natural frequency that is very close to a strong interference frequency. Consequently, the proposed adaptive method is a powerful tool to improve the accuracy of modal identification when the forced response signal has a low SNR.

\section{Acknowledgement}

The work is supported by Natural Science Foundation of China (Nos. 51421004), and the High-end CNC machine tools and fundamental manufacturing equipment key state science and technology projects (Grant No. 2012ZX04012032). 


\section{References}

394

395

396

397

398

399

400

401

402

403

404

405

406

407

408

409

410

411

412

413

414

415

416

417

418

419

420

421

422

423

424

425

426

427

428

429

430

431

432

433

434

435

1. Sanliturk KY, Cakar O: Noise elimination from measured frequency response functions. Mechanical Systems \& Signal Processing 2005; 19(3):615-631, DOI:10.1016/j.ymssp.2004.04.005.

2. Kim YY, Hong JC, Lee NY. Frequency Response Function Estimation via a Robust Wavelet DeNoising Method. Journal of Sound \& Vibration 2001; 244(4):635-649, DOI:10.1006/jsvi.2000.3509.

3. Alamdari MM, Li J, Samali B: FRF-based damage localization method with noise suppression approach. Journal of Sound \& Vibration 2014, 333(14):3305-3320, DOI:10.1016/j.jsv.2014.02.035.

4. Hu SLJ, Bao X, Li H: Model order determination and noise removal for modal parameter estimation. Mechanical Systems \& Signal Processing 2010, 24(6):1605-1620, DOI:10.1016/j.ymssp.2010.01.005.

5. Bao XX, Li CL, Xiong CB: Noise elimination algorithm for modal analysis. Applied Physics Letters 2015, 107(4):3777.DOI: 10.1063/1.4927642.

6. Randall RB: Noise and vibration data analysis. Handbook of Noise and Vibration Control 2007:549-564. DOI: 10.1002/9780470209707.ch46.

7. Randall RB: Vibration-based condition monitoring: industrial, aerospace and automotive applications: John Wiley \& Sons; 2011. DOI: 10.1002/9780470977668.

8. Ephraim Y, Malah D: Speech enhancement using a minimum-mean square error short-time spectral amplitude estimator. IEEE Transactions on Acoustics Speech \& Signal Processing 1984, 32(6):1109-1121.DOI: 10.1109/TASSP.1984.1164453.

9. Plapous C, Marro C, Scalart P: Improved Signal-to-Noise Ratio Estimation for Speech Enhancement. Audio Speech \& Language Processing IEEE Transactions on 2006, 14(6):2098-2108.DOI: 10.1109/TASL.2006.872621.

10. Imsiya KA, Nandana BT: Speech source separation and noise reduction using a MMSE short-time spectral amplitude estimator. In Innovations in Information, Embedded and Communication Systems (ICIIECS), 2015 International Conference on: 2015; 2015.DOI: 10.1109/ICIIECS.2015.7193178.

11. Abutalebi HR, Rashidinejad M: Speech enhancement based on $\beta$-order MMSE estimation of Short Time Spectral Amplitude and Laplacian speech modeling. Speech Communication 2015, 67:92-101.DOI: 10.1016/j.specom.2014.12.002.

12. Djouama A, Lim MS, Ettoumi FY: Erratum to: Channel Estimation in Long Term Evolution Uplink Using Minimum Mean Square Error-Support Vector Regression. Wireless Personal Communications 2015, 80(1):447-447. DOI:10.1007/s11277-014-2191-3.

13. Sudeep PV, Palanisamy P, Kesavadas C, Rajan J: Nonlocal linear minimum mean square error methods for denoising MRI. Biomedical Signal Processing \& Control 2015, 20:125-134. DOI: $10.1016 /$ j.bspc.2015.04.015.

14. Tiwari M, Gupta B: Image Denoising using Spatial Gradient Based Bilateral Filter and Minimum Mean Square Error Filtering. Procedia Computer Science 2015, 54:638-645.DOI:10.1016/j.procs.2015.06.074.

15. Drouet J, Leclère Q, Parizet E: Experimental modeling of Wiener filters estimated on an operating diesel engine. Mechanical Systems \& Signal Processing 2015, s 50-51:646-658.DOI: 
$436 \quad$ 10.1016/j.ymssp.2014.05.027.

437 16. Lim JS, Oppenheim AV: Enhancement and bandwidth compression of noisy speech. 438 Proceedings of the IEEE 1980, 67(12):1586-1604.DOI: 10.1109/PROC.1979.11540.

439 17. Scalart P, Filho JV: Speech enhancement based on a priori signal to noise estimation. In 440 Acoustics, Speech, and Signal Processing, IEEE International Conference on: 1996; 441 1996:629-632.DOI: 10.1109/ICASSP.1996.543199.

442 18. Martin R: Noise power spectral density estimation based on optimal smoothing and minimum 443 statistics. Speech \& Audio Processing IEEE Transactions on 2001, 9(5):504-512.DOI: $444 \quad 10.1109 / 89.928915$.

445 19. Cohen I, Berdugo B: Noise estimation by minima controlled recursive averaging for robust 446 speech enhancement. Signal Processing Letters IEEE 2002, 9(1):12-15.DOI: 10.1109/97.988717.

447 20. El-Fattah MAA, Dessouky MI, Abbas AM, Diab SM, El-Rabaie ESM, Al-Nuaimy W, 448 Alshebeili SA, El-Samie FEA: Speech enhancement with an adaptive Wiener filter. International 449 Journal of Speech Technology 2014, 17(1):53-64. DOI: 10.1007/s10772-013-9205-5.

450

451 
2

3

4

5

6

7

8

9

10

11

12

13

14

15

16

17

18

19

20

21

22

23

24

25

26

27

28

29

30

31

32

33

34

35

36

37

38

39

40

41

42

43

44

45

46

47

48

49

50

51

52

53

54

55

56

57

58

59

60

452 Table list

453

Table 1. The frequencies of the simulated signal

\begin{tabular}{ccccc}
\hline$f_{n 1}(\mathrm{~Hz})$ & $f_{n 2}(\mathrm{~Hz})$ & $f_{n 3}(\mathrm{~Hz})$ & $f_{n 4}(\mathrm{~Hz})$ & $f_{r 1}(\mathrm{~Hz})$ \\
\hline 44.0 & 50.0 & 65.0 & 80.0 & 49.5 \\
\hline
\end{tabular}

454

455

Table 2. The parameters of the simulated signal

\begin{tabular}{ccccccccccc}
\multicolumn{10}{c}{ Table 2. The parameters of the simulated signal } \\
\hline$s_{1}$ & $s_{2}$ & $s_{3}$ & $s_{4}$ & $s_{5}$ & $s_{6}$ & $s_{7}$ & $\zeta_{1}$ & $\zeta_{2}$ & $T(s)$ \\
\hline 8.00 & 14.00 & 12.00 & 13.00 & 5.90 & 0.13 & 0.16 & 10 & 6 & 6.3 \\
\hline
\end{tabular}

456

457 
458 Figure list

459 Figure 1. Classification of signals.

460 Figure 2. The actual and estimated SNRs for a simulated signal.

461 Figure 3. Flow chart of the proposed method.

462 Figure 4. Time domain waveforms of the simulated signal.

463 Figure 5. The simulated signal waveform before and after filtering.

464 Figure 6. The simulated signal spectrum before and after filtering.

465 Figure 7 The image of the generator: (a) Generator and sound-proof housing, (b) Generator shell

466 Figure 8. Bode diagram of the rotor response during startup.

467 Figure 9. Time domain waveforms of the measured signal.

468 Figure 10. The measured signal waveform before and after filtering.

469 Figure 11. The measured signal spectra before and after filtering. 


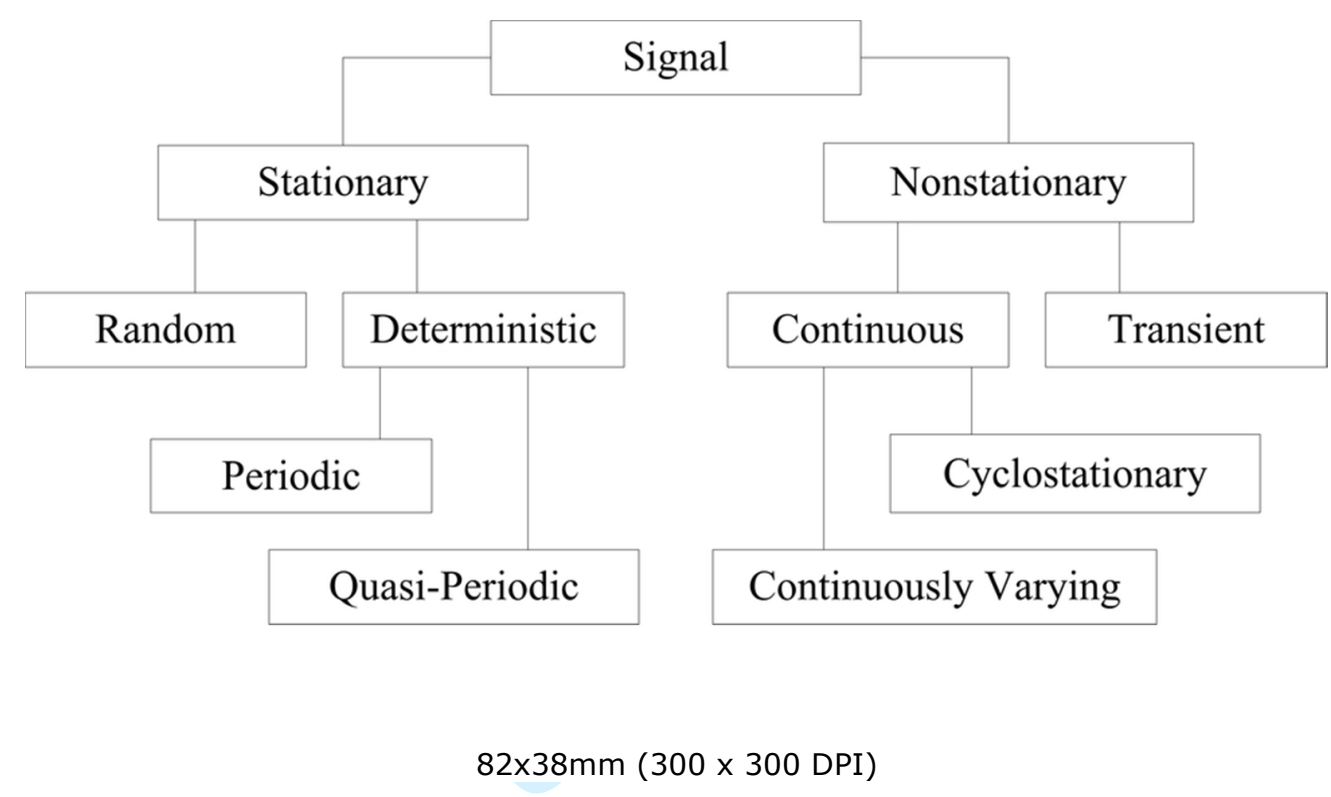




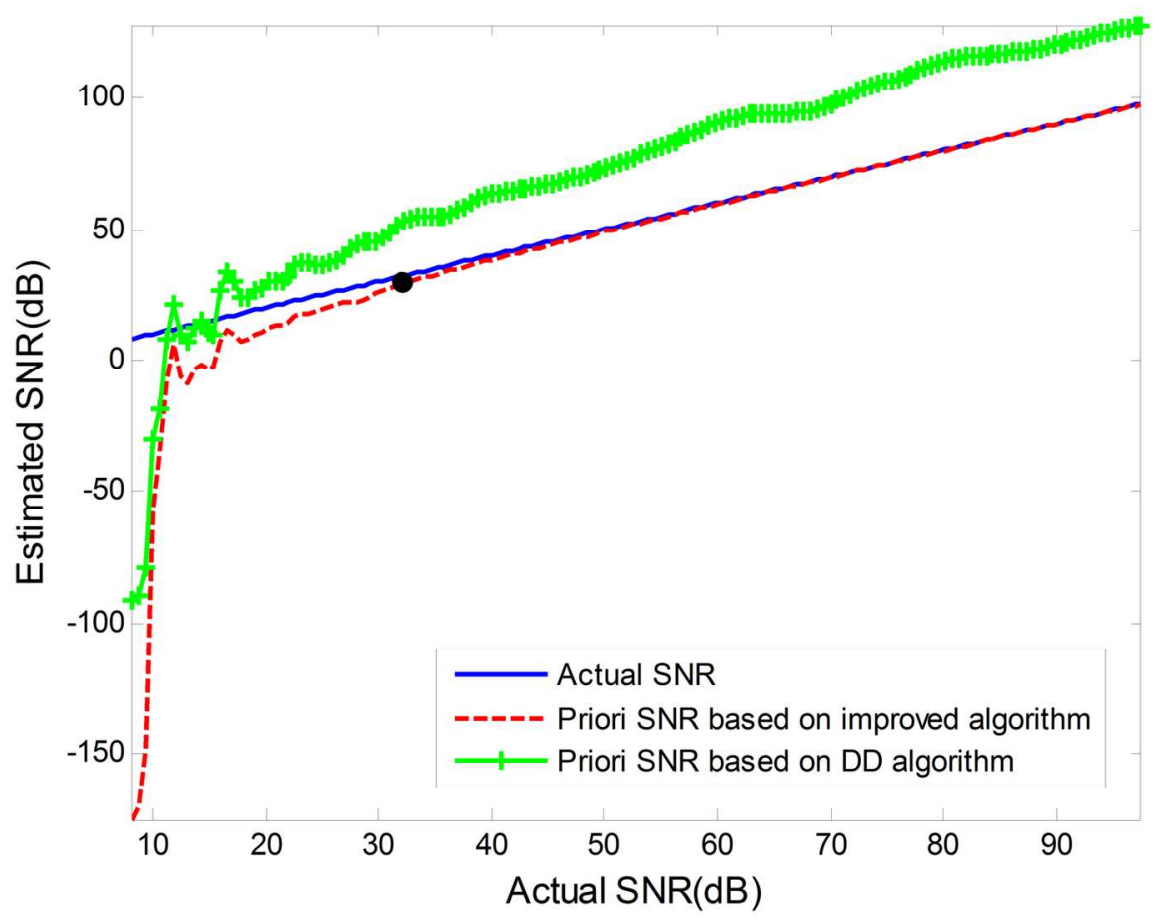

$148 \times 111 \mathrm{~mm}(300 \times 300 \mathrm{DPI})$ 


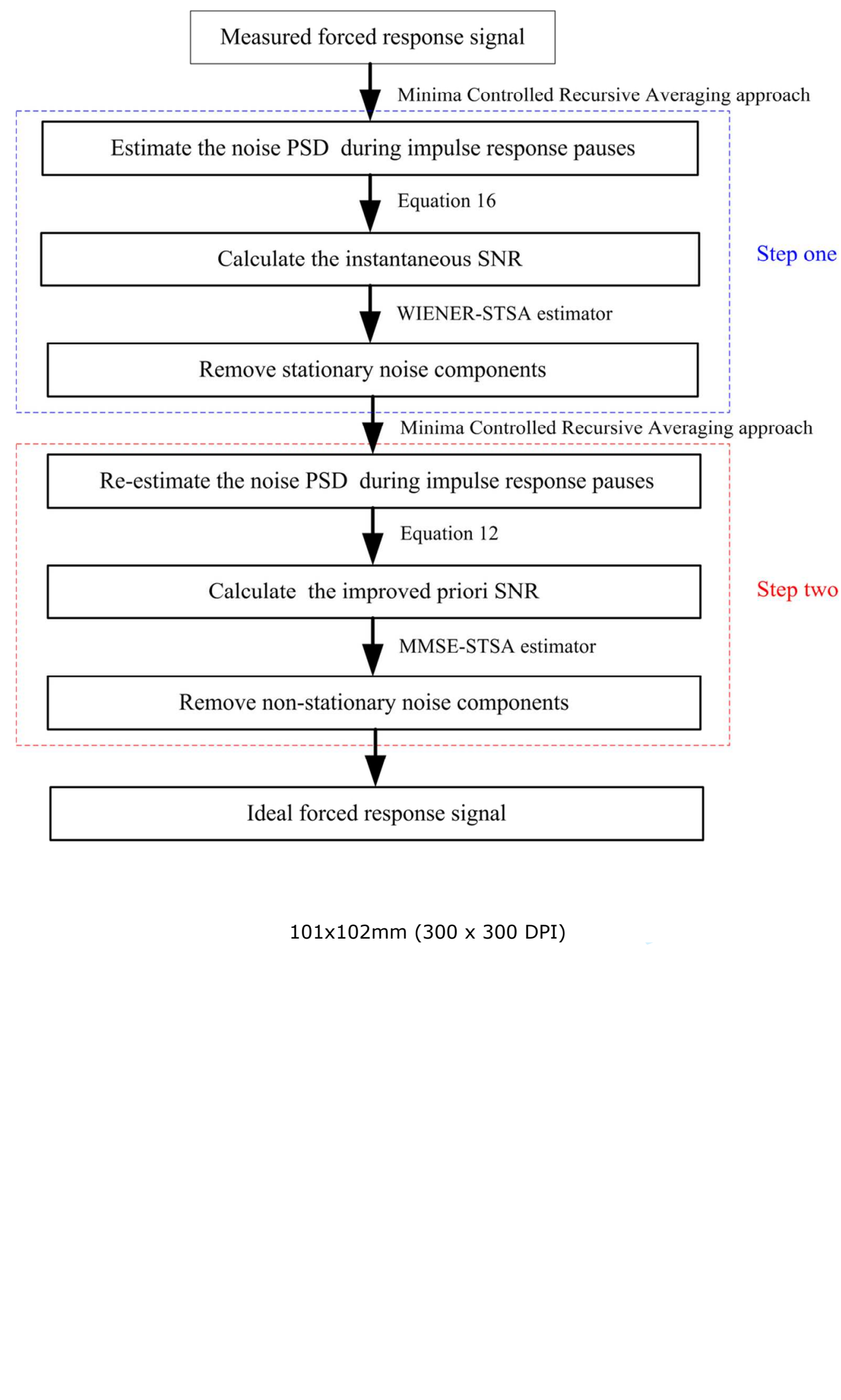




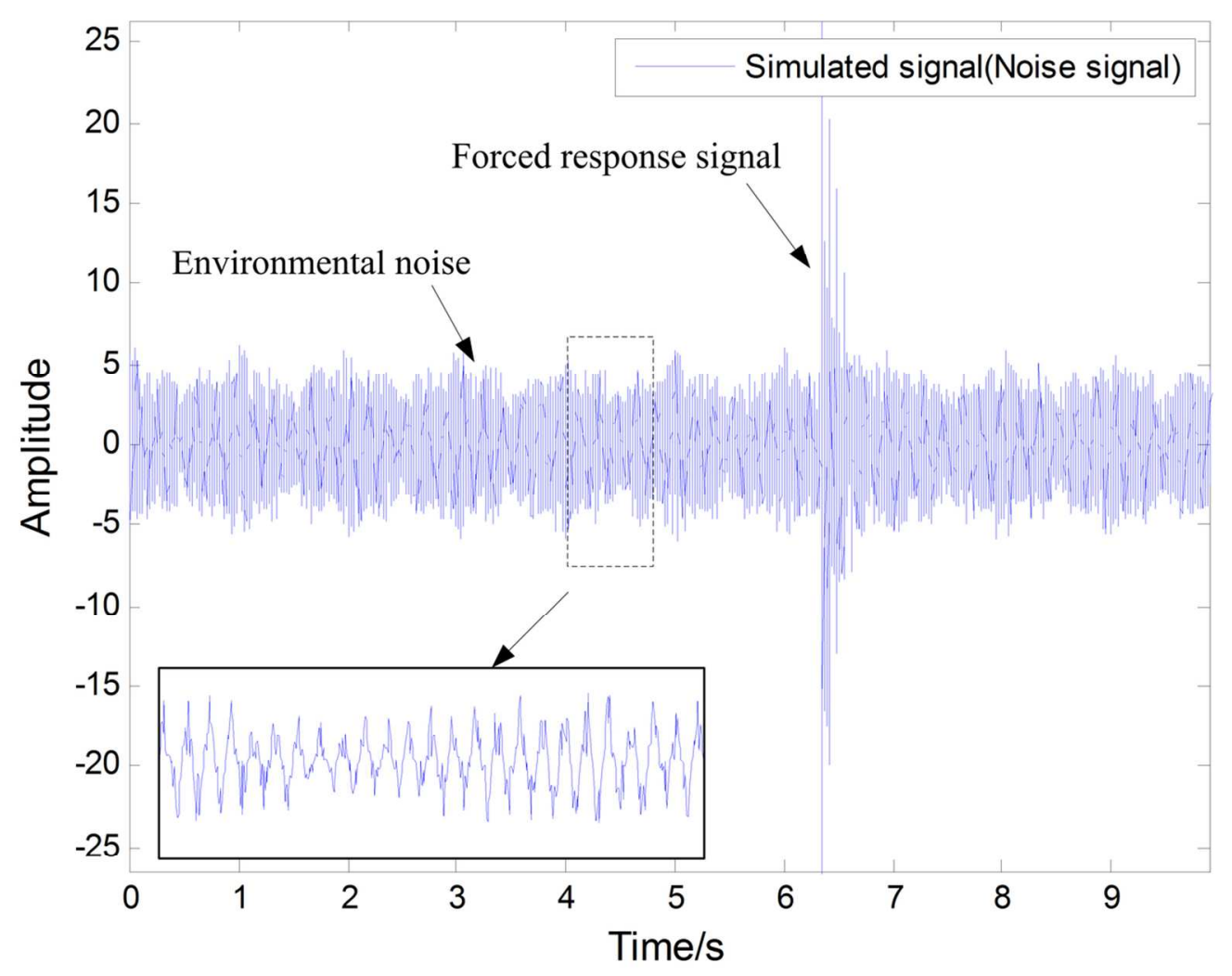

$112 \times 89 \mathrm{~mm}(300 \times 300$ DPI $)$ 


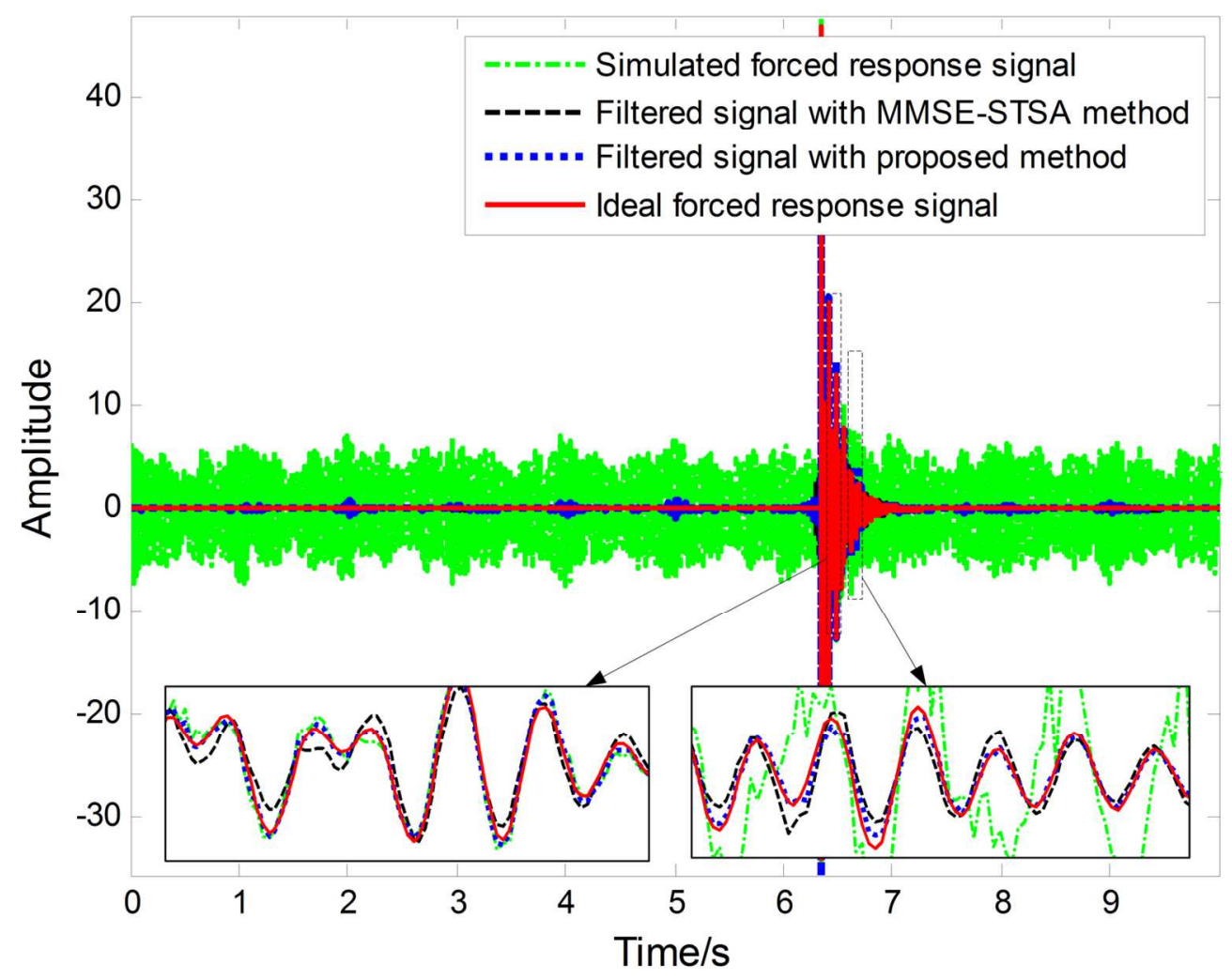

$135 \times 107 \mathrm{~mm}(300 \times 300 \mathrm{DPI})$ 


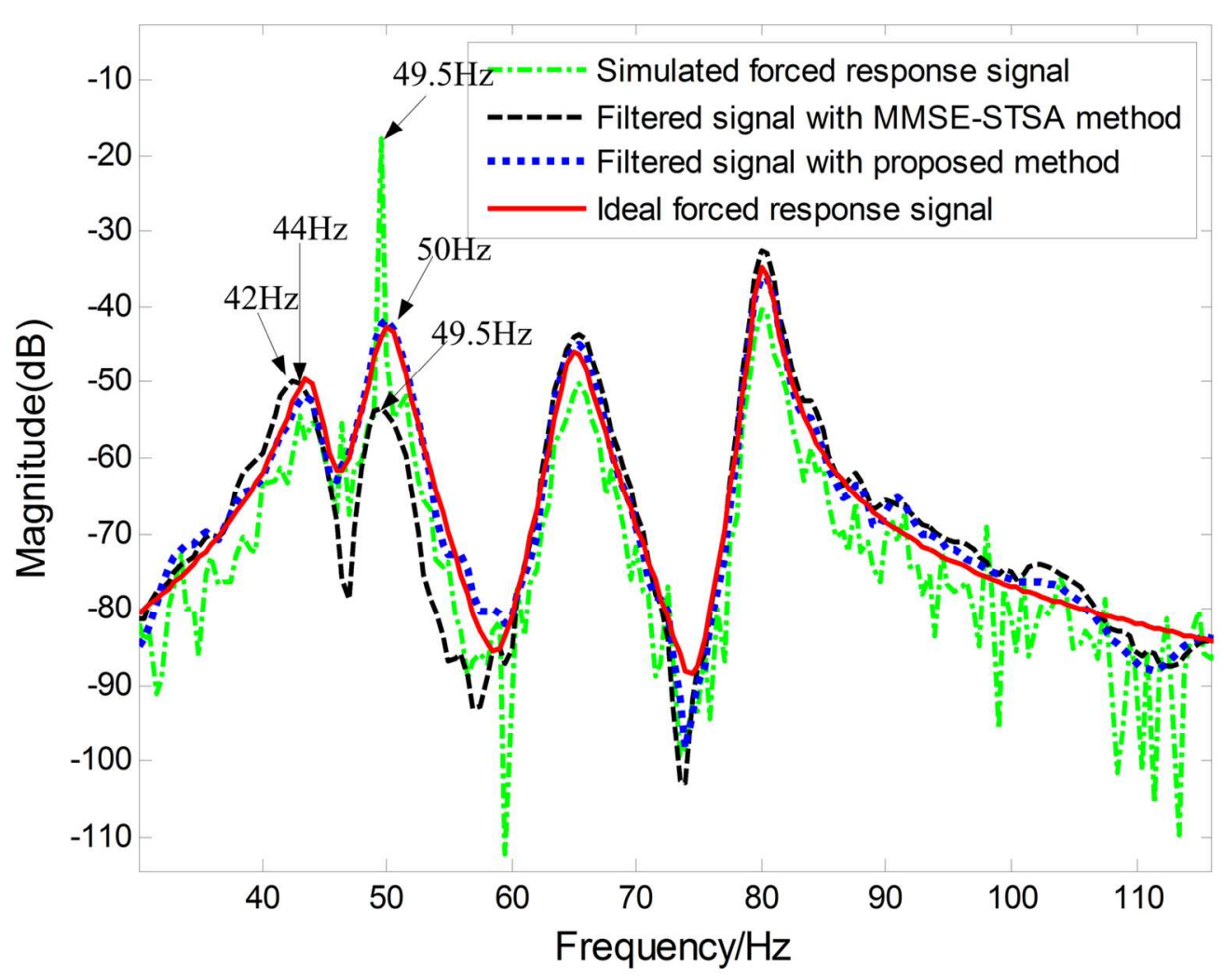

$137 \times 108 \mathrm{~mm}(300 \times 300$ DPI $)$ 


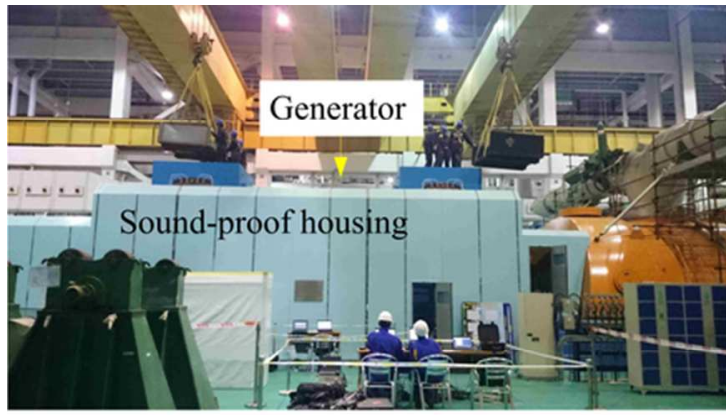

(a)

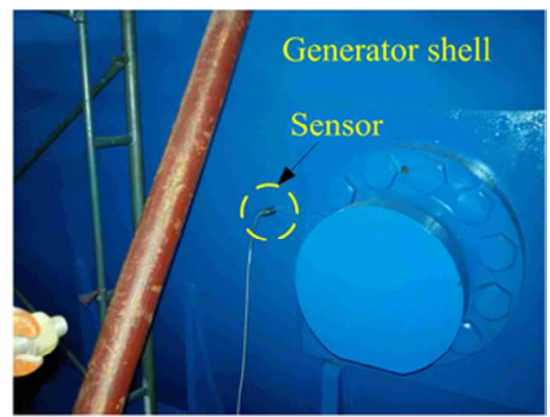

(b)

$66 \times 23 \mathrm{~mm}(300 \times 300 \mathrm{DPI})$ 

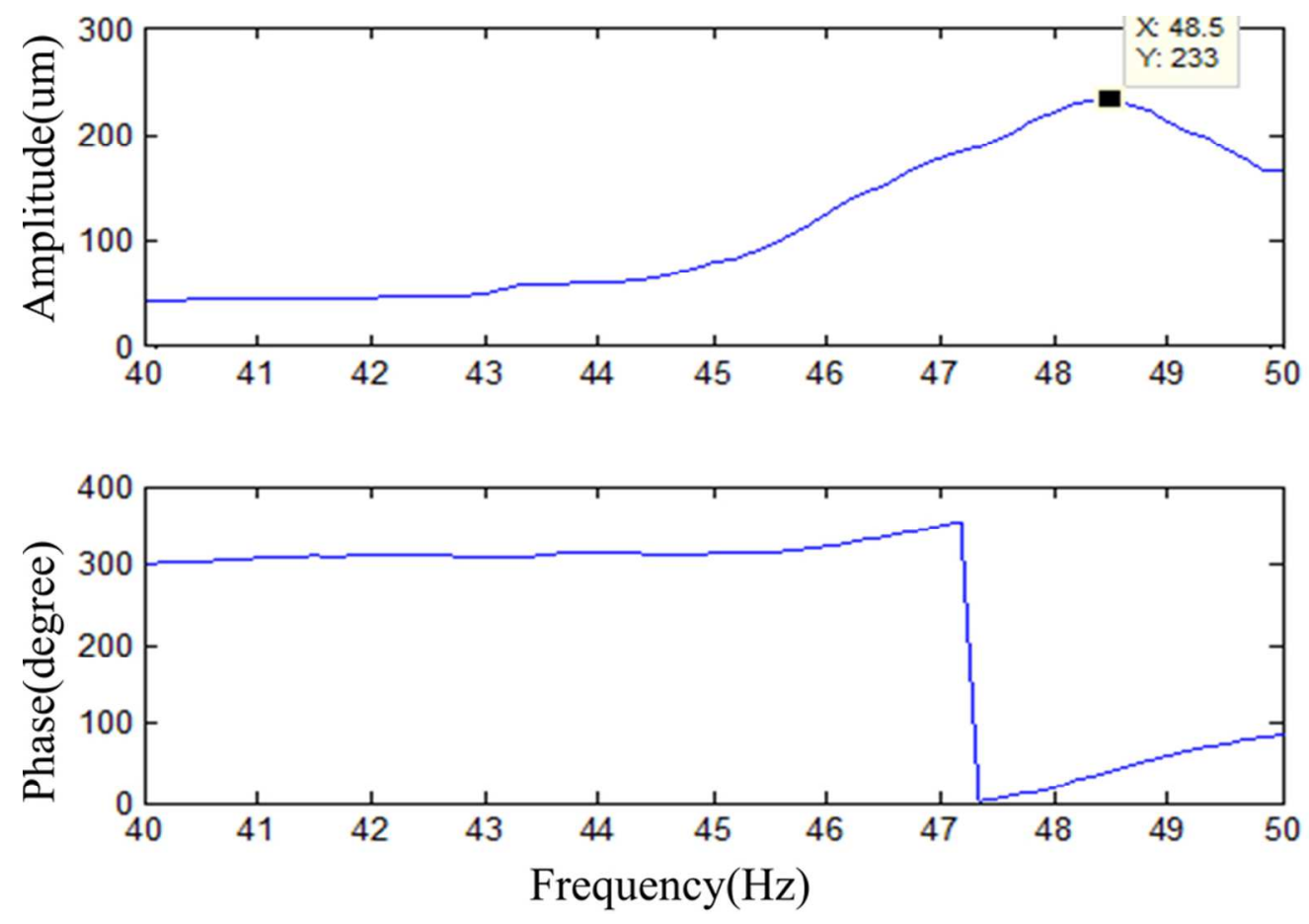

$81 \times 57 \mathrm{~mm}(300 \times 300$ DPI $)$ 
1

2

3

4

5

6

7

8

9

10

11

12

13

14

15

16

17

18

19

20

21

22

23

24

25

26

27

28

29

30

31

32

33

34

35

36

37

38

39

40

41

42

43

44

45

46

47

48

49

50

51

52

53

54

55

56

57

58

59

60

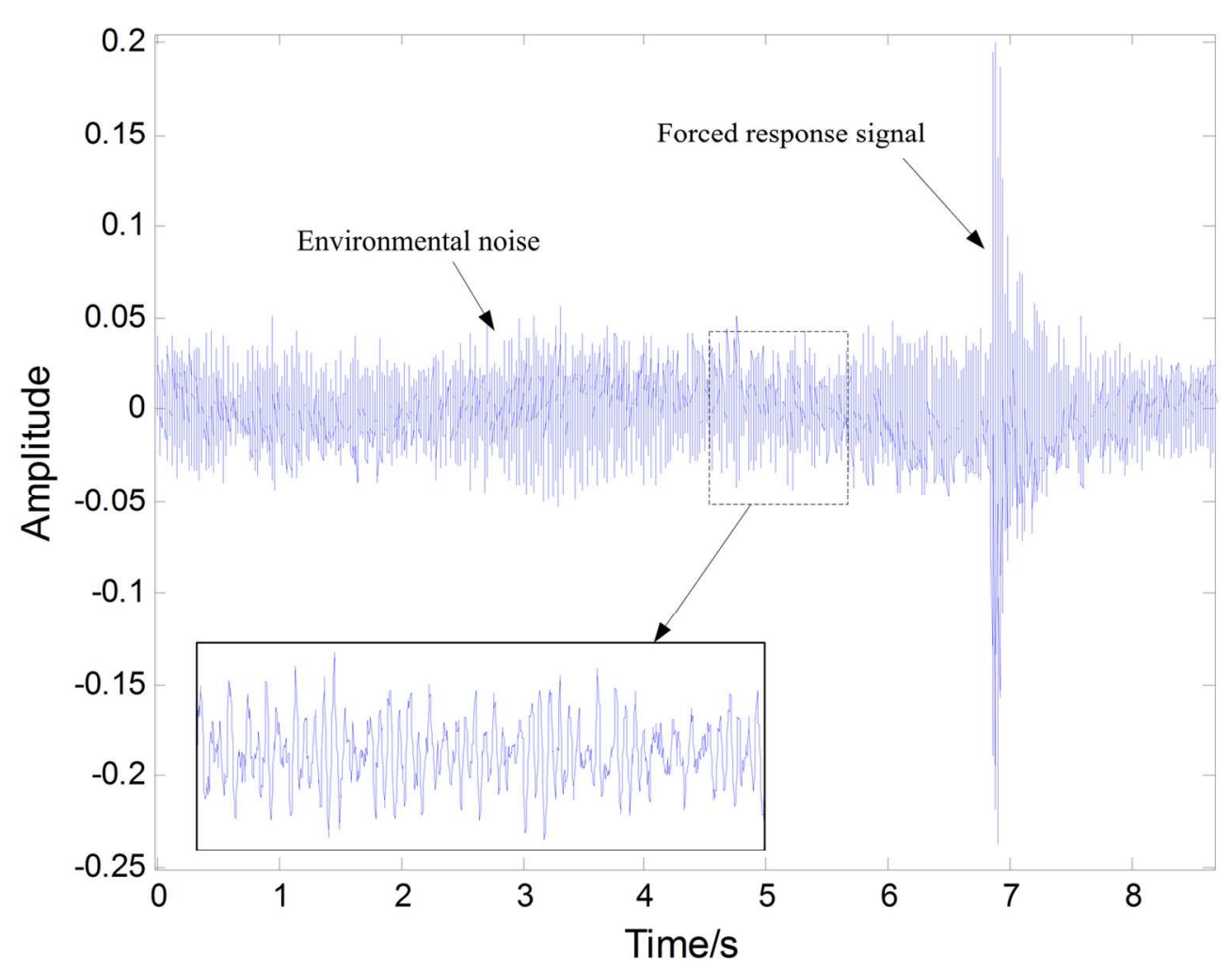

$137 \times 106 \mathrm{~mm}(300 \times 300 \mathrm{DPI})$ 


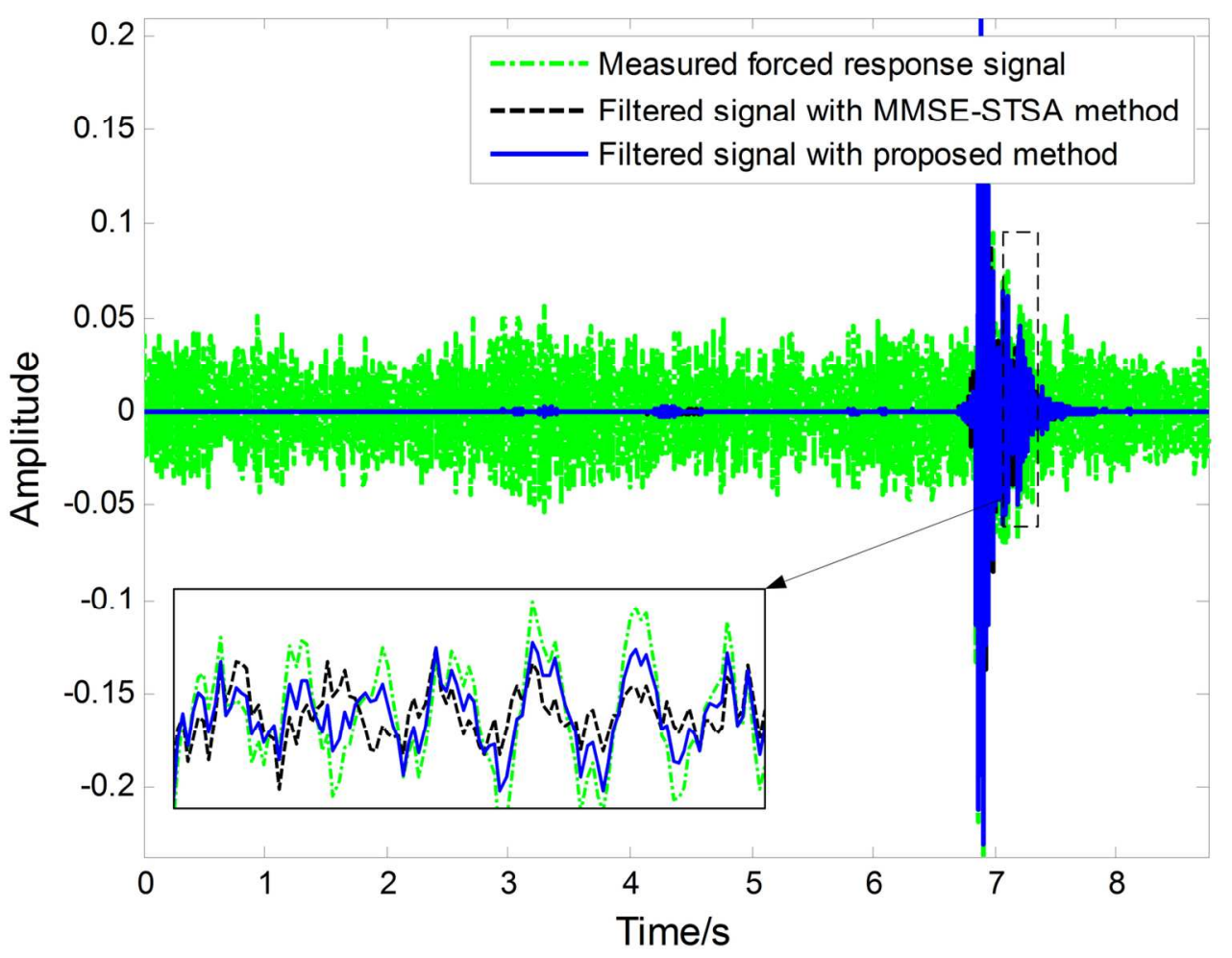

$135 \times 105 \mathrm{~mm}(300 \times 300$ DPI $)$ 


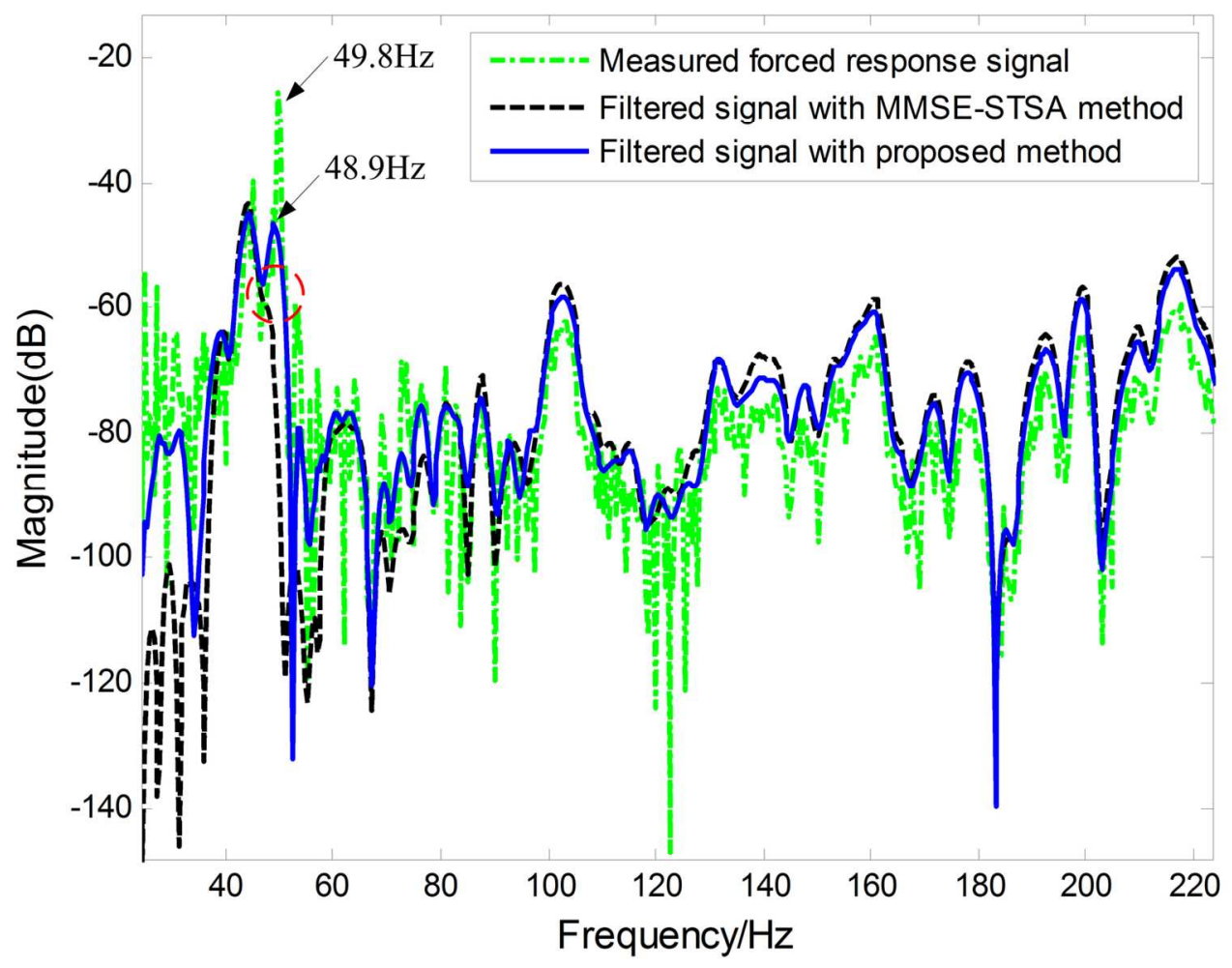

$139 \times 107 \mathrm{~mm}(300 \times 300$ DPI $)$ 